\title{
Measure and Manage the Dynamic risk of Commodity Futures Market Based on CAViaR
}

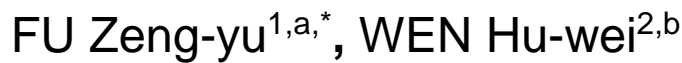 \\ ${ }^{1}$ Beijing Jiao Tong University, Haidian, Beijing, China \\ ${ }^{2}$ Huazhong University of Science \&Technology, Wuhan, Hubei, China \\ a498812316@qq.com, b564124958@qq.com
}

Keywords: Commodity Futures; CAViaR model; the family of GARCH model; Backtest.

\begin{abstract}
Under the background of OBOR strategic, commodities futures market of China meet new opportunity. It benefits the internationalization of China's futures market and enhances the commodity pricing power that we can management the market risk. By taking the typical industrial and agricultural commodities futures of the Dalian Commodity Exchange in consider, this paper adopts CAViaR model to measure the dynamic risk. Then three likelihood ratio tests and one dynamic quantile test are used to compare the predictive performance and applicability in different quantiles of different models. The empirical results show that the CAViaR model is superior to the traditional GARCH model. Yields of commodity futures presented typical "fat tail" and autocorrelation. The GARCH models, which ignore the "fat tail" and autocorrelation, may be inefficient to measure risk of commodity futures Market. The CAViaR model may be the most suitable risk management tools for commodity futures risk management.
\end{abstract}

\section{我国大宗商品期货价格风险的CAViaR测度与防范 \\ 符增昱 $1, \mathrm{a},{ }^{\star}$, 温煳炜2,b \\ 1 北京交通大学国际贸易学系，海淀，北京，中国 \\ 2华中科技大学经济学院, 武汉, 湖北, 中国 \\ a498812316@qq.com, b564124958@qq.com}

关键词：商品期货; CAViaR模型; GARCH族模型; 后测检验

中文摘要. “一带一路” 战略下中国大宗商品期货市场迎来新的发展契机, 科学有效地防范 商品期货市场风险对加速中国期货市场国际化进程、提高中国大宗商品定价权有重要意义。 本文以大连商品期货交易所工业和农业大宗商品期货的典型品种为研究对象, 建立基于 CAViaR模型的动态风险测度VaR模型, 并同时运用似然比检验和动态分位数检验对各个模型 的精准性进行后测检验, 最后研究了不同期货品种风险的动态特征与差异。结果表明, CAViaR 模型的风险预测效果要优于传统的GARCH族模型；商品期货品种呈现典型的 “高峰厚尾” 和 收益率自相关现象, 采用正态分布的GARCH族模型以及忽视收益率自相关的GARCH族模型 来防范大宗商品价格风险需要特别谨慎。在综合考虑了模型便易性、稳健性和准确性后, CAViaR模型是商品期货市场风险管理实践最为适合的风险管理工具。 


\section{1. 引言}

“一带一路”战略下，中国实体经济企业、沿线国家的市场主体利用大宗商品期货市场 管理价格风险的需求日益强烈, 有利于中国商品期货市场发展和国际化进程, 商品期货市场 迎来了新的发展契机。然而, 随着资源性产品价格形成机制改革、利率市场化改革、汇率形 成机制改革、人民币国际化战略等改革措施深层次的推进, 各类大宗商品和金融资产的价格 波动更加频繁和剧烈, 商品期货市场面临着更为频繁的国内外市场不确定因素的冲击, 因此 寻找一种行之有效的期货价格风险管理工具、科学有效地防范商品期货市场风险显得至关重 要。本文引入国际上主流的 CAViaR 方法来测度和分析中国商品期货的价格风险, 为中国监 管部门风险监管和控制、金融投资机构风险管理以及交易所保证金水平设置提供决策依据和 理论参考。

相比国内现有关于期货市场风险测度与防范的文献，本文研究特色在于，首次采用半参 数法 CAViaR 模型对商品期货市场风险度量, 并对风险趋势进行动态分析; 其次, 选取大连 商品交易所主要期货品种进行动态风险测算, 并对此几个品种的基本特征和风险防范等方面 的差异进行了对比; 最后, 样本外的后测检验不仅采用了三个似然比检验, 还加入了动态分 位数检验，克服了后测检验方法选择上的主观性。

\section{2. 动态风险测度的 CAViaR 方法}

\subsection{CAViaR 理论方法介绍}

Engle 和 Manganelli 借鉴波动率的 GARCH 建模思想, 认为 $\mathrm{VaR}$ 同样服从 GARCH 模型。 [1]既然金融资产的收益具有序列自相关性，VaR 的本质是收益率序列的某一特定分位数，那 么可以用条件自回归形式直接对 VaR 进行建模, 本文使用三种形式的 CAViaR 模型刻画价格 风险演化过程:

$$
\text { SAV-CAViaR 模型: } \quad f_{t}(\beta)=\beta_{0}+\beta_{1} f_{t-1}(\beta)+\beta_{2}\left|y_{t-1}\right|
$$

$$
\text { AS-CAViaR 模型: } \left.\quad f_{t}(\beta)=\beta_{0}+\beta_{1} f_{t-1}(\beta)\right)+\beta_{2}\left(y_{t-1}\right)^{+}-\beta_{3}\left(y_{t-1}\right)^{-}
$$

IGARCH-CAViaR 模型: $\left.\quad f_{t}(\beta)=\left(\beta_{0}+\beta_{1} f_{t-1}^{2}(\beta)\right)+\beta_{3} y_{t-1}^{2}\right)^{1 / 2}$

其中, $\left(y_{t-1}\right)^{+}$和 $\left(y_{t-1}\right)^{-}$分别表示滞后一期收益率的正部和负部, $f_{t}(\beta)$ 表示收益率的条件分 位数。方程（1）和（3）假设过去正负收益对未来风险的影响是对称的, AS-CAViaR 模型本 质上是 SAV-CAViaR 模型的一般形式，它反映了正负收益率对未来 VaR 值的非对称效应。鉴 于 AV-CAViaR 模型、AS-CAViaR 模型和 IGARCH-CAViaR 模型的的非线性和递归性，模型将 采用 Engle 等的优化方法求解, 依据 Koenker 和 Bassett 提出的分位数回归(Quantile Regression) 思想, 具体采用最小绝对离差法 (LAD) 估计。 ${ }^{[1-2]}$ LAD 法采用优化算法估计参数, 具体要 优化的目标函数为:

$$
\operatorname{Min}_{\beta} \frac{1}{T} \sum_{t=1}^{T}\left[\theta-I\left(y_{t}<f_{t}(\beta)\right)\right]\left[y_{t}-f_{t}(\beta)\right] \text {, 其中 } 0<\theta<1
$$

其中, $\theta$ 表示回归的样本分位数。相比均值回归, 它能充分刻画自变量 $\mathrm{X}$ 对于因变量 $\mathrm{Y}$ 分布的位置、刻度和形状的影响。在残差序列服从高斯分布的情况下, 分位数回归的估计效 率只有很小的损失; 针对不服从正态分布的时间序列, 特别是对具有“尖峰厚尾”性质的金融 收益序列，它能准确地捕捉到分布的尾部特征，估计结果将更为稳健性。

\section{2 后测检验方法}

为了检验和比较上述 CAViaR 模型、GARCH 族模型以及 EVT 模型的动态 VAR 预测精度, 
本文选择 RQ 值, DQ 检验, 以及 $L R$ 统计量作为模型后测检验方法。 RQ 值即目标函数公式 （4），当 RQ 值越小，表示拟合效果越好，是衡量模型优劣的一个标准。DQ 检验和 LR 统 计量检验定义在击中事件的基础上，当 $y_{t}<-V a R_{t}$ 时也就是 $\mathrm{VaR}$ 不足以覆盖实际损失，称之 为一次击中 Hit 事件。据此, 定义 “击中序列” 如下:

$$
\text { hit }_{t}=I\left(y_{t}<-V a R_{t}\right)
$$

其中, $\mathrm{I}$ 是示性函数，当 $y_{t}<-V a R_{t}$ 时， I 取值为 1 ; 当 $y_{t} \geq-V a R_{t}$ 时, $\mathrm{I}$ 取值为 0 。根据 Kupiec(1995) 的研究结果, 如果该模型能有效地预测市场风险, 那么该 “击中序列” 应服从 贝努利分布。[3]

\subsection{1 似然统计量检验方法}

$\mathrm{LR}$ 统计量检验包含非条件覆盖统计量 $\mathrm{LR}_{\mathrm{uc}}$ 以及独立性统计量 $\mathrm{LR}_{\text {ind }}$ 和条件覆盖统计量 $\mathrm{LR}_{\mathrm{cc}}, \mathrm{LR}_{\mathrm{uc}}$ 考虑击中事件的发生概率, $\mathrm{LR}_{\mathrm{ind}}$ 考虑击中事件发生的独立性, 条件覆盖统计量 $\mathrm{LR}_{\mathrm{cc}}$ 是 $\mathrm{LR}_{\mathrm{uc}}$ 和 $\mathrm{LR}_{\mathrm{ind}}$ 的综合, 同时考虑击中事件发生概率和发生相依性, 为了节约篇幅, 不做过多 描述，具体可以参考志宏（2015）。[4]

2.2.2 动态分位数检验

Engle 和 Manganelli 提出 CAViaR 模型时同时提出了动态分位数检验, DQtest 统计量服从 自由度为 $p+n+2$ 的卡方分布:

$$
\begin{gathered}
\text { DQhit }_{\theta, t}=\text { hit }_{i t}-\theta=I\left(y_{t}<-V a R_{t}\right)-\theta \\
\text { DQhit }_{\theta, t}=\beta_{0}+\sum_{i=1}^{p} \beta_{i} D Q h i t_{\theta, t-\mathrm{i}}+\beta_{p+1} V_{a R_{t}}+u_{t} \\
\text { DQtest }=\frac{\delta_{\mathrm{ols}} X^{\prime} X \delta_{\text {ols }}}{\theta(1-\theta)} \rightarrow \chi^{2}(K)
\end{gathered}
$$

其中, $\theta$ 为给定的分位数, 当 $y_{t}<-V a R_{t}$ 时, $D Q h i t_{\theta, t}=1-\theta$; 当 $y_{t} \geq-V a R_{t}$ 时, $D Q h i t_{\theta, t}=-\theta$ 。如果模型参数估计正确, 那么 $E\left(D Q h i t_{\theta, t} \mid \Omega_{\mathrm{t}-1}\right)=0$ 。这说明 $D Q h i t_{\theta, t}$ 对于任何 滞后的 $D Q h i t_{\theta, t-\mathrm{k}}$ 以及预测的 $V a R_{t-k}$ 都应不具备相关性，即回归方程（7）总体应该不显著。

\section{3. 大商所商品期货市场的典型事实}

\section{1 样本选择和数据处理}

综合考虑不同商品期货品种中的重要性、期货合约的成交量大小以及上市交易时间等因 素后，本文选择了大连商品期货交易所的豆粕期货、聚氯乙烯期货价格指数为实证样本，价 格指数以每天的结算价作为该天的期货价格。样本区间从聚氯乙烯期货上市时间后新的一年 开始, 即从 2008 年 1 月 7 日到 2016 年 10 月 28 日，其中后 500 个数据样本用于样本外的检 验。数据来源于 Wind 数据库的南华期货价格指数, 选择该指数是因为连续合约和主力合约 价格指数均会因为合约变换带来异常值, 导致收益率涨跌幅太大，按成交量加成、按持仓量 加成的价格指数也均无法避免这一问题，而南华期货价格指数对这一问题进行了修正。

\section{2 收益率分布的典型事实}

图 1 和表 1 给出了大连商品期货交易所两种代表性商品期货收益率的波动情况和 QQ 图 法检验收益率是否正态分布。从图 1 可以看出, 两种商品期货市场场均存在明显的波动聚集 现象，金融资产价格的波动率聚集这一典型事实是大量 $\mathrm{GARCH}$ 模型发展的前提，本文的 CAViaR 建模也是以波动率聚集的典型事实为前提。图 2 检验则表明聚氯乙烯期货和豆粕期货 
的收益率并不服从正态分布，具有高峰后尾分布，商品期货具有较大的尾部风险。

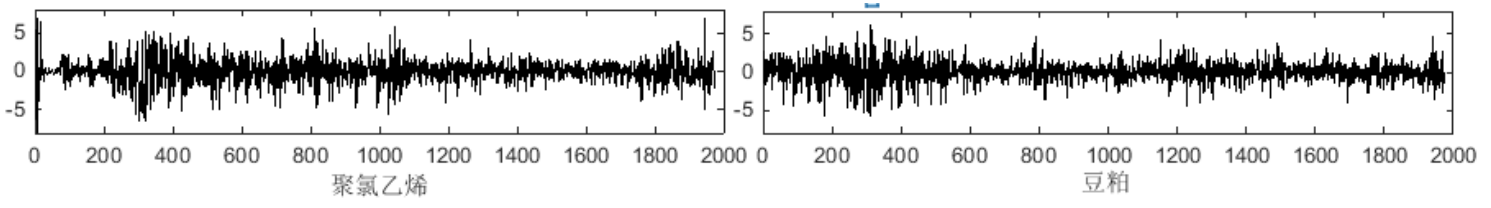

图 1 两种商品期货的收益波动状况
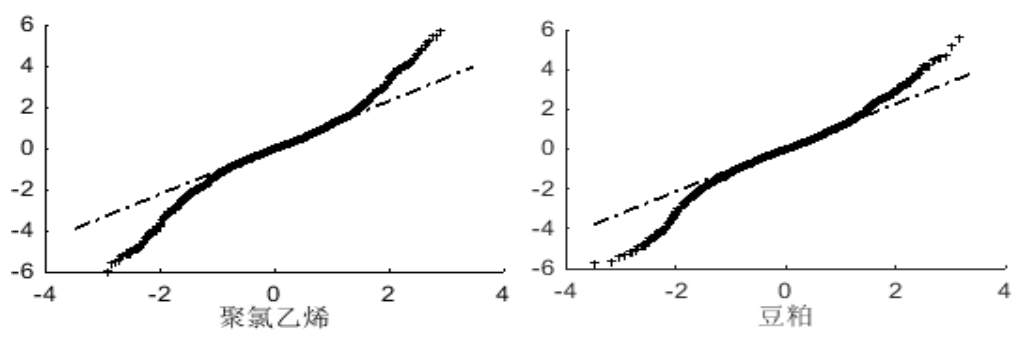

图 2 两种商品期货的收益率的 $\mathrm{QQ}$ 图

表 1 是对收益率的描述性统计分析。偏度、峰度和 JB 检验一致表明所有商品期货的收益 率均不服从正态分布，普遍呈现出显著的 “有偏” 和 “尖峰” 形态; ARCH 效应检验结果表明 两个期货指数均存在波动聚集效应，适合采用 GARCH 模型进行拟合，CAViaR 建模的典型事实 的基础存在; LBQ 统计量说明收益率存在典型的相关性, 从而说明收益率存在相关性, GARCH 族建模过程中需要考虑收益率的 ARMA 形式, 即遵循 ARMA-GARCH 建模思路, 给 GARCH 族建模 带来不少困难, 然而 CAViaR 建模思路可以有效地避免收益率自相关带来的建模困难; ADF 统 计值则表明所有的收益序列显著拒绝存在单位根的原假设, 即聚氯乙烯指数和豆粕指数的收 益序列均是平稳的。

表 1 大连商品交易所两种商品期货收益率的描述性统计

\begin{tabular}{lccllllll}
\hline & 均值 & 标准差 & 偏度 & 峰度 & JB 值 & ARCH 值 & LBQ 值 & ADF 值 \\
\hline 聚氯乙烯 & -0.00017 & 0.0155 & $-0.2833^{*}$ & $5.9331^{*}$ & $734.39^{*}$ & $50.89^{*}$ & $388.27^{*}$ & $-44.50^{*}$ \\
豆粕指数 & 0.00042 & 0.0140 & $-0.2210^{*}$ & $4.9369^{*}$ & $324.78^{*}$ & $21.10^{*}$ & $278.91^{*}$ & $-46.29^{*}$ \\
\hline
\end{tabular}

注: *表示在 $1 \%$ 水平下显著。

\section{4. 实证结果和分析}

\subsection{CAViaR 模型的估计结果}

表 2 给出了豆粕和聚氯乙烯这两个期货品种的 CAViaR 模型在 $1 \%$ 和 $5 \%$ 的分位数下的参数 估计结果和 $\mathrm{DQ}$ 检验结果。首先, 无论是 $1 \%$ 的分位数还是 $5 \%$ 的分位数, 样本内 $\mathrm{DQ}$ 检验在 $10 \%$ 的显著性水平下均无法拒绝原假设, 说明三个 CAViaR 模型对商品期货市场的尾部风险拟合效 果都非常好; 其次, 从样本外 DQ 检验可以看出, CAViaR 测算的 $1 \% \mathrm{VaR}$ 和 $5 \% \mathrm{VaR}$ 都经得起后 测检验, 除了聚氯乙烯的 AS-CAViaR 模型 DQ 检验的 P 值较小, 其它均在 $10 \%$ 的显著性水平下 均无法拒绝原假设, 说明 CAViaR 模型能够良好地刻画我国期货市场的尾部风险特征, 具有预 测尾部风险的功能; 最后, $1 \% \mathrm{VaR}$ 的 $\mathrm{DQ}$ 检验 $\mathrm{P}$ 值普遍大于 $5 \% \mathrm{VaR}$ 的 $\mathrm{P}$ 值检验, 非常接近于 1 , 说明 CAViaR 模型在刻画极端风险时具有很大优势。

CAViaR 模型的回归系数大部分是统计意义上显著的, 说明波动率的 GARCH 效应和 ARCH 效应显著存在, AS-CAViaR 模型的结果显示, 除 1\%分位数下的豆粕期货外, Beta3 均小于 Beta4, 说明大宗商品期货市场存在杜杆效应, 利空消息引起的波动大于利好消息所引起的波动。值 得注意的是, 虽然回归结果部分参数在统计意义上不显著的, 但这些变量依然是不可或缺的: 
一是为了保持该模型与以往模型的内在连贯性; 二是省略其中一些不显著的变量均会导致模 型的预测效果降低。三个 CAViaR 模型虽然都比较好的刻画了商品期货的尾部风险, 但是 AS-CAViaR 模型在 5\%VaR 的测算上相对较有优势; 无论是在 $1 \% \mathrm{VaR}$ 测算上还是在 $5 \% \mathrm{VaR}$ 测算 上, AS-CAViaR 和 IGARCH-CAViaR 效果不相伯仲, RQ 值和 DQ 检验的 P 值都比较理想并难以区 分优劣，相较之下，SAV-CAViaR 模型对商品期货的风险刻画效果则稍逊一筹。

表 2 CAViaR 模型估计结果和 DQ 检验

\begin{tabular}{|c|c|c|c|c|c|c|}
\hline & \multicolumn{2}{|c|}{ SAV-CAViaR } & \multicolumn{2}{|c|}{ AS-CAViaR } & \multicolumn{2}{|c|}{ IGARCH-CAViaR } \\
\hline & 聚氯乙烯 & 豆粕 & 聚氯乙烯 & 豆粕 & 聚氯乙烯 & 豆粕 \\
\hline \multicolumn{7}{|l|}{$1 \% \mathrm{VaR}$} \\
\hline \multirow{2}{*}{ Beta1 } & $0.211^{*}$ & $0.064^{* * *}$ & 0.489 & $0.036^{*}$ & 0.562 & $0.080^{* *}$ \\
\hline & $(0.133)$ & $(0.025)$ & $(0.529)$ & $(0.024)$ & $(0.687)$ & $(0.048)$ \\
\hline \multirow{2}{*}{ Beta2 } & $0.917^{* * * *}$ & $0.971^{* * *}$ & $0.797^{\text {**** }}$ & $0.968^{* * *}$ & $0.877^{* * *}$ & $0.974^{* * * *}$ \\
\hline & $(0.049)$ & $(0.013)$ & $(0.160)$ & $(0.018)$ & $(0.049)$ & $(0.004)$ \\
\hline \multirow{2}{*}{ Beta3 } & $0.172^{*}$ & $0.064^{* * * *}$ & 0.132 & $0.104^{*}$ & 0.704 & 0.134 \\
\hline & $(0.112)$ & $(0.026)$ & $(0.169)$ & $(0.065)$ & $(2.128)$ & $(0.248)$ \\
\hline \multirow{2}{*}{ Beta4 } & - & - & $0.389^{* *}$ & $0.045^{* *}$ & - & - \\
\hline & - & - & $(0.209)$ & $(0.025)$ & - & - \\
\hline RQ 值 & 76. 032 & 60.434 & 75.301 & 59.033 & 75.292 & 60.527 \\
\hline 样本内 DQ & 0.352 & 0.328 & 0.272 & 0.981 & 0.288 & 0.279 \\
\hline 样本外 DQ & 0.000 & 0.989 & 0.974 & 0.838 & 0.988 & 0.995 \\
\hline \multicolumn{7}{|l|}{$5 \% \mathrm{VaR}$} \\
\hline \multirow{2}{*}{ Beta1 } & $0.136^{* * *}$ & $0.064^{\text {**** }}$ & $0.123^{*}$ & $0.037^{*}$ & $0.132^{*}$ & 0.028 \\
\hline & $(0.054)$ & $(0.022)$ & $(0.080)$ & $(0.023)$ & $(0.097)$ & $(0.048)$ \\
\hline \multirow{2}{*}{ Beta2 } & $0.890^{* * * *}$ & $0.936^{* * * *}$ & $0.860^{* * *}$ & $0.901^{* * *}$ & $0.879^{* * *}$ & $0.915^{* * *}$ \\
\hline & $(0.043)$ & $(0.027)$ & $(0.049)$ & $(0.019)$ & $(0.023)$ & $(0.014)$ \\
\hline \multirow{2}{*}{ Beta3 } & $0.192^{* * *}$ & $0.144^{* * *}$ & $0.149^{* *}$ & $0.124^{* * *}$ & $0.274^{* * *}$ & $0.258^{* * *}$ \\
\hline & $(0.044)$ & $(0.050)$ & $(0.067)$ & $(0.046)$ & $(0.295)$ & $(0.231)$ \\
\hline \multirow{2}{*}{ Beta4 } & - & - & $0.225^{* * *}$ & $0.239^{* * *}$ & - & - \\
\hline & - & - & $(0.063)$ & $(0.041)$ & - & - \\
\hline RQ 值 & 259.691 & 238.359 & 257.599 & 236.334 & 257.607 & 237.490 \\
\hline 样本内 DQ & 0.230 & 0.259 & 0.668 & 0.607 & 0.855 & 0.494 \\
\hline 样本外 DQ & 0.005 & 0.237 & 0.109 & 0.422 & 0.163 & 0.207 \\
\hline
\end{tabular}

注: *、**、林表示 $1 \% 、 5 \% 、 10 \%$ 显著性水平下显著; $\mathrm{DQ}$ 检验报告 $\mathrm{P}$ 值; 括号内数字代表对应参数的标准差。

\section{2 动态 VaR 测度的似然比检验与模型比较}

由于 CAViaR 模型在测算极端尾部风险时（1\%VaR）效果非常好，本文只将 CAViaR 模型与 GARCH 族模型比较, 旨在说明 CAViaR 模型在对商品期货风险刻度的准确性远高于普通的 GARCH 族模型，尽管穷尽 GARCH 族模型可能能够找到更优的模型，但从风险管理的实践角度来说， 模型的稳健性和便易性是一个重要的考量, CAViaR 模型更适应商品期货市场的风险管理建模。 从表 3 似然比检验结果来看, 三种 CAViaR 模型风险度量的准确性都较高, 并且 AS 模型和 IGARCH 模型风险测算准确性稍高于 SAV 模型。CAViaR 模型刻画风险的准确性远高于 GJR 和 AR-GARCH-t 模型 ${ }^{\mathbb{1}}$ 。表 1 的 LBQ 检验已经说明, 商品期货收益率存在非常显著的自相关性, 而忽视这一相关性的 GJR 模型对风险刻度效果都十分差, 非条件覆盖统计量 $\mathrm{LR}_{\mathrm{uc}}$ 的 P 值近似 等于 0 , 考虑了收益自相关和厚尾分布的 AR-GARCH-t 模型测算准确性有所改善, 但不如 CAViaR

\footnotetext{
(1) 笔者还对多个基础的 GARCH 族模型进行了商品期货 VaR 值的似然比检验, 结果准确率都不高。
} 
模型。GARCH 族众多模型的选择往往是困难的, 收益率方程的假定和分布的假定进一步增加 了 GARCH 模型建模困难, CAViaR 模型很好的避免了这一问题。EVT 模型对数据量的苛求往往 难以得到满足, CAViaR 模型在度量极端尾部风险表现又十分良好, 相较于其它 GARCH 族和 EVT 族模型，CAViaR 更适合金融风险管理实践。

表 3 各种动态 VaR 测度模型的似然比检验

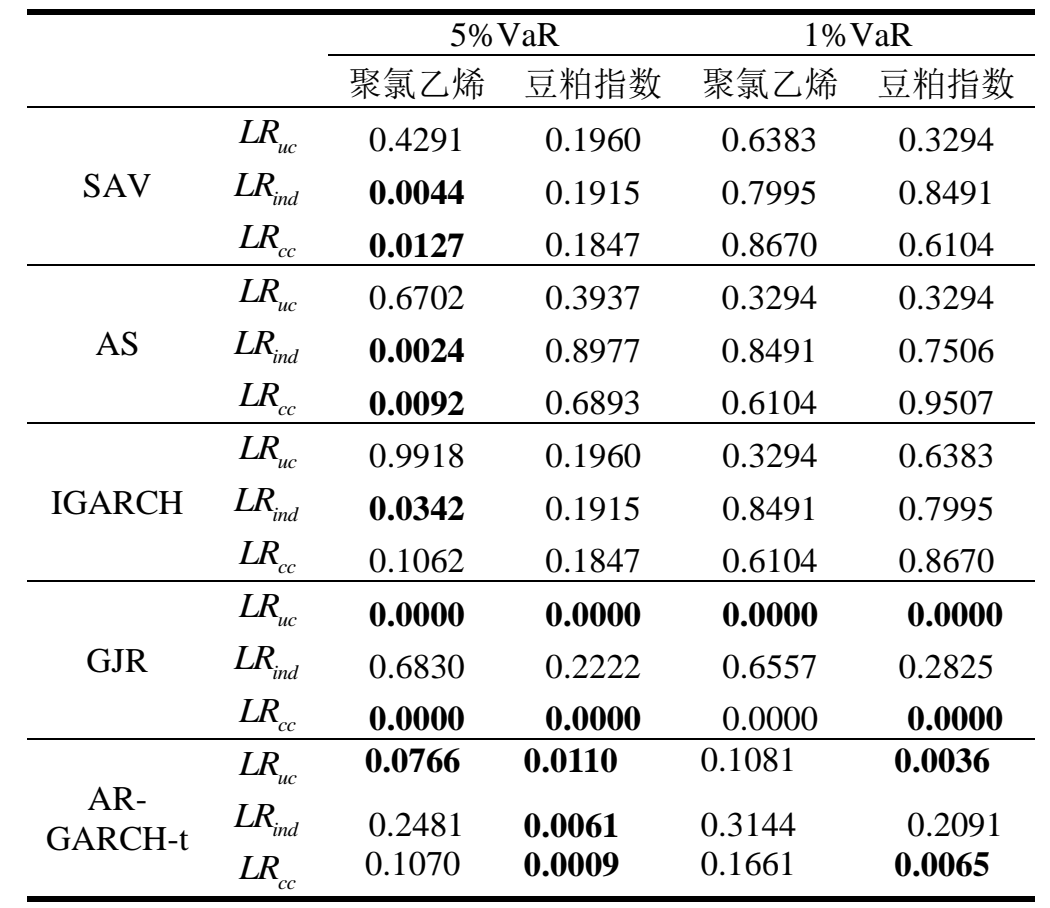

\section{5. 结束语}

本文以大连商品期货交易所两个典型代表的工业和农业大宗商品期货品种为研究对象, 采 用半参数法 CAViaR 模型的测度我国大宗商品价格风险, 并运用似然比检验和动态分位数检验 对各个模型的精准性进行后测检验, 最后研究了不同期货品种风险的动态特征与差异。本文 结果表明: (1) 三种 CAViaR 模型对大宗商品期货市场的风险预测准确性都非常高, 模型建立 也简便，模型预测准确度稳健性高，相较于其它 GARCH 族和 EVT 族模型, CAViaR 更适合金融 风险管理实践; (2) 三种 CAViaR 模型模型中, AS 模型和 IGARCH 模型风险测算准确性稍高 于 SAV 模型, 商品期货市场呈现 “杜杆效应” 的特征, 但是, 这一特征并不明显; (3) 商品 期货品种呈现典型的 “高峰厚尾” 和收益率自相关现象, 收益率序列不服从正态分布, 因此 投资者使用正态分布假设下的 GARCH 族模型以及忽视收益率自相关的 GARCH 族模型来防范大 宗商品价格风险需要特别谨慎。CAViaR 模型不需要假定收益率的分布, 也不需要设定收益率 方程, 能有效地减少模型误设的风险, 本文严谨的后测检验方法也证实了 CAViaR 模型要比 GARCH 族模型具有更优异的风险预测能力。

\section{References}

[1] Engle R. F., Manganelli S.. CAViaR: Conditional Autoregressive Value at Risk by Regression Quantile[J]. Journal of Business and Economic Statistics, 2004, 22(4):367-381.

[2] Kupiec P H. Techniques for verifying the accuracy of risk measurement models[J]. Journal of Derivatives, 1995, 3(2):73-84.

[3] YU Bai-min, WU Wei-xing. VaR Forecast Comparison between Realized Volatility ARFI and CAViaR Models[J]. Chinese Journal of Management Science, 2015, 23(2):50-58. 
[4] JIAN Zhi-hong, PENG Wei. The Overnight Risk of Exchange Rate Research Based on CAViaR[J]. Chinese Journal of Management Science, 2015, 06:17-24.

[5] WANG Peng, WANG Hong, WEI Yu. Research on Stylized Facts and Risk Models for Chinese Agricultural Futures Market[J]. Journal of Industrial Engineering/ Engineering Management, 2013, 27(3):172-182.

[6] ZHANG Ying, ZHANG Fu-xiang. The Theory and Empirical Research of Quantile Regression in Financial Risk Measurement[J]. The Journal of Quantitative \& Technical Economics, 2012, 04:95-109. 